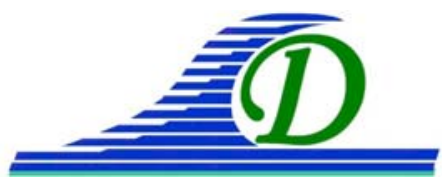

XIII ${ }^{\text {èmes }}$ Journées Nationales Génie Côtier - Génie Civil

Dunkerque, 2-4 juillet 2014

DOI:10.5150/jngcgc.2014.063 C Editions Paralia CFL

disponible en ligne - http://www.paralia.fr - available online

\title{
Estimation des volumes d'eau contenus dans les lacs de faible superficie par télédétection multi-satellitaire et mesures in situ : exemple du lac la Bure
}

\author{
Frédéric FRAPPART $^{1,2}$, Frédéric BAUP ${ }^{3}$, Jérôme MAUBANT ${ }^{1,3}$, \\ Vincent MARIEU ${ }^{4}$, Alexandra SPODAR ${ }^{4}$, \\ Jean-Paul PARISOT ${ }^{4}$, Guillaume DETANDT ${ }^{4}$
}

1. GET, UMR 5563, OMP, 31400 Toulouse, France.

frederic.frappart@get.obs-mip.fr

2. LEGOS, UMR 5566, OMP, 31400 Toulouse, France.

3. CESBIO, UMR 5126, OMP, 32000 Auch, France.

4. EPOC, UMR 5805, OASU, 33405 Talence, France.

\section{Résumé :}

A l'heure actuelle, le suivi des ressources en eau se fait au moyen des trois techniques: les réseaux de mesure in situ, la modélisation hydrologique, et l'observation satellitaire. Etant données la diminution drastique du nombre de mesures hydrologiques disponibles à court-terme et les difficultés rencontrées pour modéliser l'ensemble des réservoirs hydrologiques à grande échelle, le suivi des ressources en eau par télédétection satellitaire a connu un essor rapide. Le suivi des eaux de surface nécessite de déterminer les volumes stockés dans les lacs, fleuves et plaines d'inondation. Des techniques combinant observations satellitaires de la hauteur, de la surface et des relevés in situ ont permis de déterminer les volumes et variations de volumes d'eau dans des grands lacs et des grands bassins fluviaux. L'objectif de notre étude est de montrer que des techniques similaires sont applicables aux réservoirs agricoles de plus faible superficie ( $<60 \mathrm{ha}$ ). Nous présentons trois méthodes utilisant les estimations de superficie inondées par imagerie satellitaire à haute résolution, de niveau d'eau par altimétrie satellitaire et de mesures in situ de niveau d'eau et de volume pour déterminer les volumes d'eau et leurs variations dans des lacs d'irrigation situés à l'ouest de Toulouse. Une bathymétrie des lacs sera effectuée au moyen d'un sondeur mono-faisceau et permettra de valider nos estimations.

Mots-clés : Imagerie haute résolution, Altimétrie radar, Lac, Bathymétrie, Volume d'eau.

\section{Introduction}

Les problèmes d'accès aux ressources en eau sont devenus un enjeu majeur sous les effets conjugués de la variabilité climatique, de l'accroissement démographique, de l'urbanisation et de l'industrialisation (OECD, 2012). L'irrigation des cultures représentant $70 \%$ de la consommation d'eau dans le monde, une bonne gestion des 
ressources en eau est nécessaire pour la mise en place de pratiques agricoles durables (YOUNGER, 2012). Les ressources en eau sont évaluées de trois manières différentes : au moyen des mesures in situ, par modélisation hydrologique, et par télédétection satellitaire. Etant données la chute du nombre de réseaux de mesures à travers le monde et les difficultés rencontrées pour modéliser l'ensemble des réserves mondiales en eau, être capable de mesurer depuis l'espace les niveaux des eaux de surface est devenu un enjeu pour les hydrologues dans les années à venir (ALSDORF et al., 2007).

Des études récentes ont montré qu'il est possible d'estimer les variations de volume d'eau de surface par combinaison d'images satellitaires et de mesures d'altimétrie radar (FRAPPART et al., 2012; DUAN \& BASTIAANSSEN, 2013). Des techniques similaires n'ont pour l'instant jamais été appliquées à des lacs de faible superficie.

Dans cette étude, nous proposons trois méthodes différentes pour estimer le volume d'eau contenu dans des lacs à partir d'images satellitaires à haute résolution spatiale, de mesures d'altimétrie radar et de données in situ. Notre but est de démontrer l'applicabilité de ces techniques à des lacs de faible superficie ( $<60 \mathrm{ha})$. Nous les utiliserons pour déterminer les variations de volumes d'eau du lac la Bure, un petit réservoir dont la superficie maximale est de 52 ha et situé dans le Sud-Ouest de la France, à proximité de Toulouse.

\section{Zones d'étude et données}

\subsection{Lac la Bure}

Le lac la Bure (4324'54" N; $1^{\circ} 09^{\prime} 07^{\prime \prime}$ E) est situé dans le sud-ouest de la France, à 40 $\mathrm{km}$ au sud-est de Toulouse. C'est un réservoir artificiel creusé en 1987 pour l'irrigation des cultures avoisinantes. Son bassin versant, d'une superficie de $20,70 \mathrm{~km}^{2}$ et dont la pente moyenne est de $2,7 \%$, est recouvert de champs (40,90\%), de forêts (24,02\%), de prairies $(33,40 \%)$ et de retenues d'eau (1,68\%). Sa surface peut atteindre 52 ha et son volume 4 millions de $\mathrm{m}^{3}$. Son remplissage et sa vidange sont dus au ruissellement des eaux de pluie tout au long de l'année, à l'évaporation et aux pompages pour l'irrigation ayant lieu principalement en été (BAUP et al., 2012). Il est géré par le Syndicat Intercommunal d'Aménagement Hydraulique de la vallée du Touch et de ses affluents (SIAH). Il situé sous la trace 773 de l'altimètre RA-2 à bord du satellite ENVISAT et dans l'emprise au sol de trois imageurs à haute résolution spatiale embarqués sur les satellites Formosat-2, TerraSAR-X, et Radarsat-2.

\subsection{Les données satellitaires}

Les données satellitaires utilisées ici sont de deux sortes: des images à haute-résolution spatiale (HR) et des hauteurs altimétriques. Quarante-cinq images à haute-résolution ont été acquises en 2010, trente-trois d'entre elles dans le domaine des micro-ondes actives par les radar à synthèse d'ouverture (SAR) à bord de TERRASAR-X, un satellite du 


\section{XIII ${ }^{\text {èmes }}$ Journées Nationales Génie Côtier - Génie Civil \\ Dunkerque, 2-4 juillet 2014}

Centre Aérospatial Allemand (DLR) faisant des acquisitions à une résolution spatiale d'au mieux 3 m en mode StripMap (BREIT et al., 2010), et de Radarsat-2, un satellite de l'Agence Spatiale Canadienne (CSA) faisant des acquisitions à une résolution spatiale de $3 \mathrm{~m}$ in Ultra Fine Mode (MORENA et al., 2004), les douze autres par l'imageur multispectral à bord du satellite Formosat-2, un satellite de l'Organisme Spatial National Taïwanais (NSPO) faisant des acquisitions à une résolution spatiale de 8 m (CHERN et al., 2008). Les images SAR ont été calibrées, géoréférencées au moyen d'images aéroportées de l'IGN à la résolution spatial de $50 \mathrm{~cm}$ pour obtenir une géolocalisation précise à moins de $6 \mathrm{~m}$, et filtrées des effets de chatoiement. Les images multi-spectrales ont été orthorectifiées et corrigées des effets atmosphériques (SAND et DE BOISSEZON, 2006) et des nuages (HAGOLLE et al., 2010). Les mesures altimétriques ont été acquises entre février 2002 et octobre 2010 par l'altimètre RA-2 à bord du satellite ENVISAT de l'Agence Spatiale Européenne (ESA), fonctionnant en bande $\mathrm{Ku}(f=13,575 \mathrm{GHz})$ et ayant une résolution spatiale le long de la trace de $350 \mathrm{~m}$, et une répétitivité temporelle de 35 jours (ZELLI, 1999). Elles sont distribuées par le Centre de Topographie de l'Océan et de l'Hydrosphère (CTOH - http://ctoh.legos.obsmip.fr/).

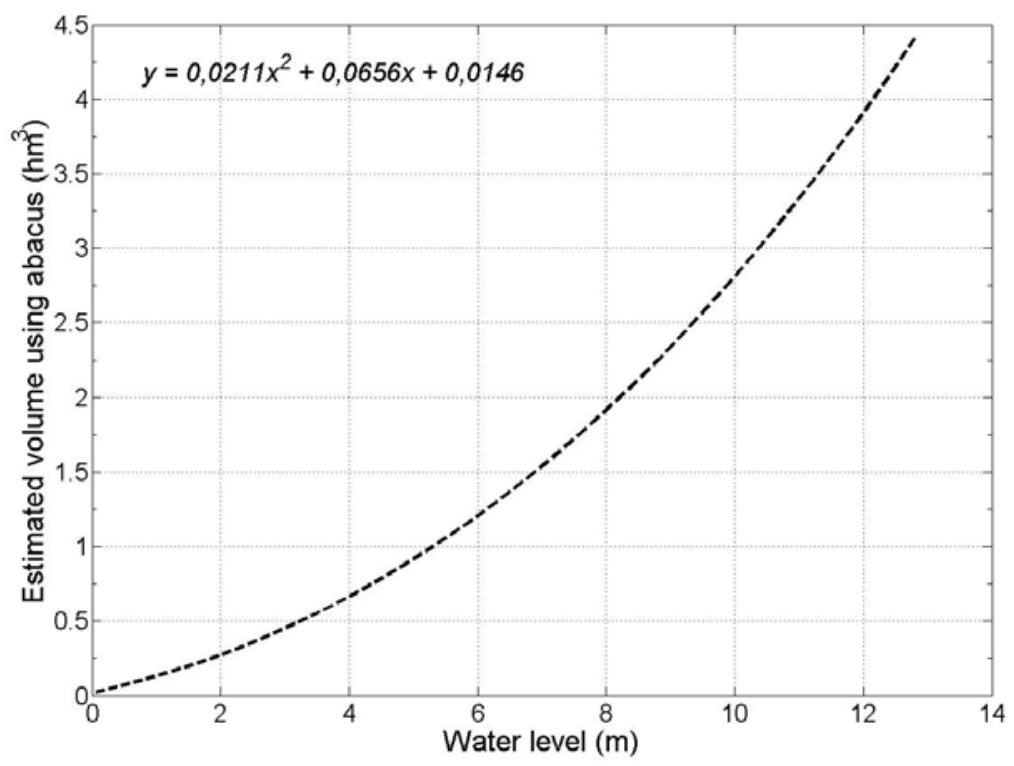

Figure 1. Courbe reliant la hauteur au volume du lac la Bure. Cette relation a été établie en 1987 lors de la construction du lac.

\subsection{Les données in situ}

Les mesures de pluie proviennent d'un pluviomètre de Météo-France situé à moins de 5 $\mathrm{km}$ du centre du lac. Les niveaux d'eau du lac sont mesurés automatiquement chaque semaine par des capteurs de pression, et lus sur une règle chaque mois. La relation entre 
la hauteur et le volume du lac a été établie en 1987 lors de la construction du lac (figure 1). Un sondage bathymétrique réalisé en 2010 par l'Agence de l'Eau AdourGaronne a permis de retrouver la même relation à $2 \%$ près. Les différences observées peuvent s'expliquer par les erreurs de chacune de deux méthodes et un léger envasement du lac.

\section{Méthodes}

\subsection{Superficie du lac par imagerie haute résolution}

Une classification parallélépipédique automatique a été appliquée à chacune des images haute-résolution pour déterminer quels pixels correspondent à de l'eau, suivant la procédure décrite par RICHARDS (1999). Les pixels correspondant à de l'eau ont ensuite été vectorisés pour en déduire la bordure du lac pour chaque image. Quelle que soit la source des images (Formosat-2, Radarsat-2 ou TerraSAR-X), les estimations sont cohérentes entre elles, la surface du lac variant de 42 à 52 ha au cours de l'année 2010. La différence maximale entre deux estimations consécutives à moins de 5 jours d'intervalle ne dépasse jamais les 4 ha, soit un écart-quadratique moyen de 8,5\% de la surface moyenne du lac et est en moyenne de 0,51 ha, soit $1,1 \%$ de la surface du lac. L'évolution temporelle de la bordure du lac est présentée en figure 2 pour 4 images acquises par Radarsat-2.

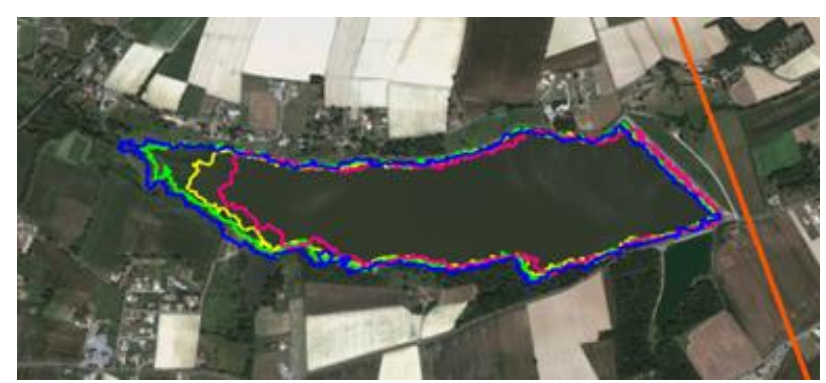

Figure 2. Evolution temporelle des berges du lac en 2010 : 31/05 (vert), 23/06 (jaune), 30/09 (rose), 11/11 (bleu). La ligne orange représente la trace au sol théorique de l'altimètre RA-2 à bord d'ENVISAT.

\subsection{Niveaux d'eau altimétriques}

Les hauteurs sont déduites des mesures altimétriques comme la différence entre l'altitude du satellite sur son orbite, la distance entre le satellite-sol déduite du temps d'aller-retour de l'onde électromagnétique émise au nadir et des corrections instrumentales, de propagation et géophysiques (voir FRAPPART et al., 2006). Elles sont exprimées par rapport à l'ellipsoïde WGS84. Les niveaux du plan d'eau sont ensuite obtenus par une sélection précise des hauteurs altimétriques correspondant à la surface du lac (entre deux et cinq à chaque passage du satellite) pour obtenir une 


\section{XIII ${ }^{\text {èmes }}$ Journées Nationales Génie Côtier - Génie Civil \\ Dunkerque, 2-4 juillet 2014}

hauteur moyenne du lac lors de chaque survol du lac entre février 2002 et octobre 2010 (figure 3). Pour une description détaillée du processus de sélection des mesures valides, se reporter à SANTOS DA SILVA et al. (2010).

\subsection{Volumes d'eau du lac}

Trois approches indépendantes ont été développées pour déterminer le volume du lac et ses variations temporelles. Les deux premières méthodes reposent sur l'estimation d'une relation empirique entre la surface du lac et sa hauteur obtenue par télédétection et des estimations de volume déduites des mesures in situ de hauteur proches dans le temps. Il est ensuite possible d'appliquer cette loi pour obtenir le volume du lac soit à partir des estimations de surface par imagerie HR, soit à partir des hauteurs altimétriques. La troisième est basée sur la combinaison des surfaces par imagerie HR et des hauteurs par altimétrie satellitaire acquises à quelques jours d'intervalle pour déterminer des variations de volume $\Delta V$ entre les temps $t 1$ et $t 2$ au moyen de l'équation (1), suivant la méthode proposée par TAUBE (2000) :

$\Delta V=S(t 1)|\Delta H|+\operatorname{sgn}(\Delta H) \frac{|\Delta S| \Delta H \mid}{2}$

où : $\Delta H=H(t 2)-H(t 1)$ est la variation de niveau d'eau entre $t 2$ et $t 1$ et $\Delta S=S(t 2)-S(t 1)$, la variation de surface du lac.

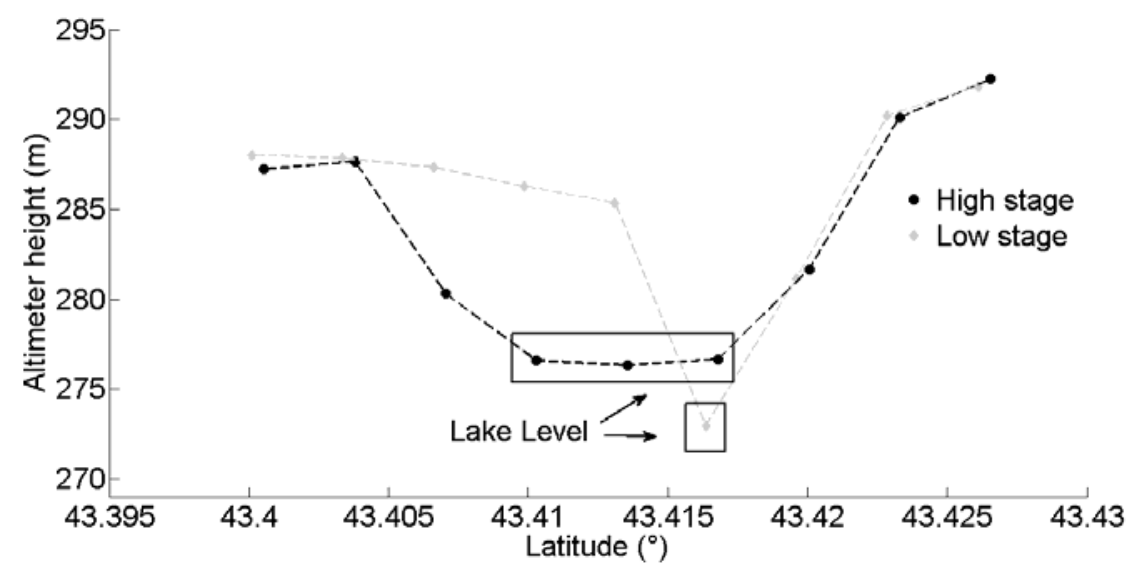

Figure 3. Variations de la hauteur altimétrique le long de la trace au sol.

\section{Résultats}

\subsection{Volume du lac par imagerie HR}

Les variations temporelles de volume du lac la Bure ont été obtenues au moyen d'une formule empirique reliant les surfaces obtenues par imagerie haute résolution et les estimations de volumes dérivés des hauteurs d'eau in situ en 45 dates communes. Elles sont présentées sur la figure 4. Un bon accord est obtenu entre les deux estimations de 
volume d'eau du lac, caractérisé par un coefficient de détermination de 0,90 et une erreur quadratique moyenne de $0,2 \mathrm{hm}^{3}$ sur les estimations de volume, soit $7,4 \% \mathrm{du}$ volume moyen au cours de l'année 2010.

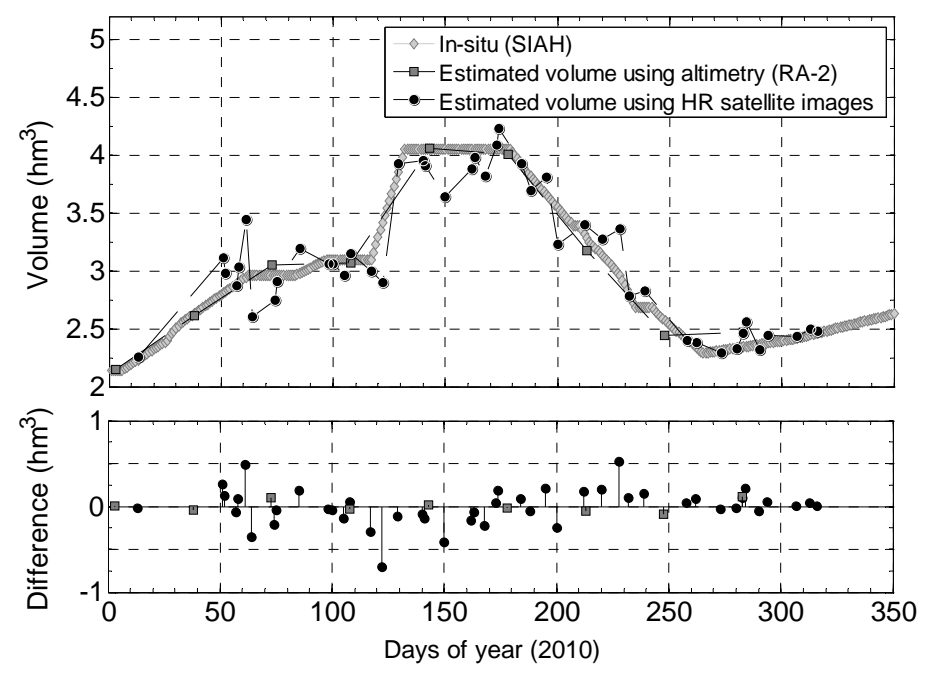

Figure 4. Evolution temporelle des volumes d'eau contenus dans le lac en 2010 (haut) estimations du SIAH (losanges gris clair), par altimétrie (carrés gris foncé) et par imagerie HR (ronds noirs) - et des erreurs associées (bas).

\subsection{Validation des niveaux altimétriques du lac}

Les estimations de niveau d'eau issues de l'altimétrie satellitaire ont été validées par comparaison aux mesures in situ effectuées par le SIAH entre 2002 et 2010. Les différences observées ne dépassent pas les $0,3 \mathrm{~m}$ dans plus de $77 \%$ des cas et n'atteignent qu'en une unique occasion les $0,75 \mathrm{~m}$ (figure 5). L'écart quadratique moyen et le coefficient de détermination des deux séries valent respectivement $0,27 \mathrm{~m}$ et 0,99 , montrant le très bon accord entre les deux sources de données. Les variations saisonnières (maxima en mai-juin et minima en septembre-octobre) et interannuelles (minimum au cours de la sécheresse de 2003) sont bien restituées par les mesures altimétriques en dépit de la faible répétitivité d’ENVISAT. Ces résultats sont comparables à ceux obtenus sur des lacs de plus grandes superficies (RICKO et al., 2012; DUAN \& BASTIAANSSEN, 2013) ou sur des grands fleuves (FRAPPART et al., 2006; SANTOS DA SILVA et al., 2010). 


\section{XIII ${ }^{\text {èmes }}$ Journées Nationales Génie Côtier - Génie Civil \\ Dunkerque, 2-4 juillet 2014}

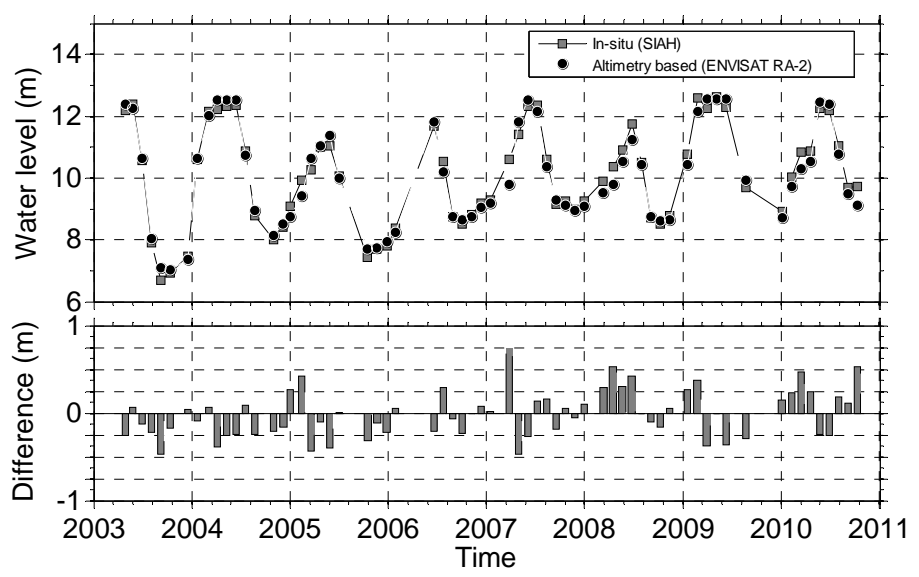

Figure 5. Evolution temporelle des niveaux d'eau du lac la Bure de 2003 à 2010 (haut) - mesures in situ (carrés gris), par altimétrie (ronds noirs) - et des erreurs associées (bas).

\section{3 $\underline{\text { Volume du lac par altimétrie }}$}

De même qu'en 4.2, une formule empirique reliant volumes provenant des mesures in situ et hauteurs d'eau issues de l'altimétrie a été établie sur 65 points entre 2003 et 2010. Les résultats sont présentés sur la figure 4 pour 2010. Un très bon accord est obtenu entre les deux estimations avec un coefficient de détermination égal à 0,97 , un écart quadratique moyen de $0,14 \mathrm{hm}^{3}$, soit $5,3 \%$ en relatif.

\subsection{Variations de volume du lac par combinaison d'imagerie HR et d'altimétrie}

Les variations de volume ont été calculées à partir de l'équation (1) pour 5 dates en 2010 pour lesquelles l'intervalle de temps entre les acquisitions des images HR et des mesures altimétriques soit inférieur à 5 jours $(\Delta V / V<0,7 \%)$. Elles ont été comparées aux estimations de variations de volume in situ. Les résultats suivants ont été obtenus : $\mathrm{R}^{2}=$ 0,98 et $\mathrm{RMSE}=0,06 \mathrm{hm}^{3}$. Ils sont meilleurs que ceux obtenus avec l'imagerie $\mathrm{HR}\left(\mathrm{R}^{2}=\right.$ 0,95 et $\left.\mathrm{RMSE}=0,17 \mathrm{hm}^{3}\right)$ ou l'altimétrie radar $\left(\mathrm{R}^{2}=0,90\right.$ et $\left.\mathrm{RMSE}=0,25 \mathrm{hm}^{3}\right)$ et les données in situ aux mêmes dates (figure 6).

\section{Discussion}

Dans l'optique d'une amélioration de la gestion de la ressource en eau, il est crucial de pouvoir estimer les quantités d'eau continues dans les petits lacs. Seule la télédétection sera en mesure éventuellement en mesure de le faire au niveau mondial. Les résultats présentés plus haut démontrent ce potentiel suivant trois approches. Les deux premières peuvent être utilisées lorsque les réservoirs sont situés sous la trace altimétrique ou dans l'empreinte au sol d'une image HR, et que des données in situ sont disponibles (ce qui est rare à l'échelle du globe). La résolution temporelle de l'imagerie HR étant plus élevée que celle de l’altimétrie, elle permet un suivi plus régulier des lacs et réservoirs. 

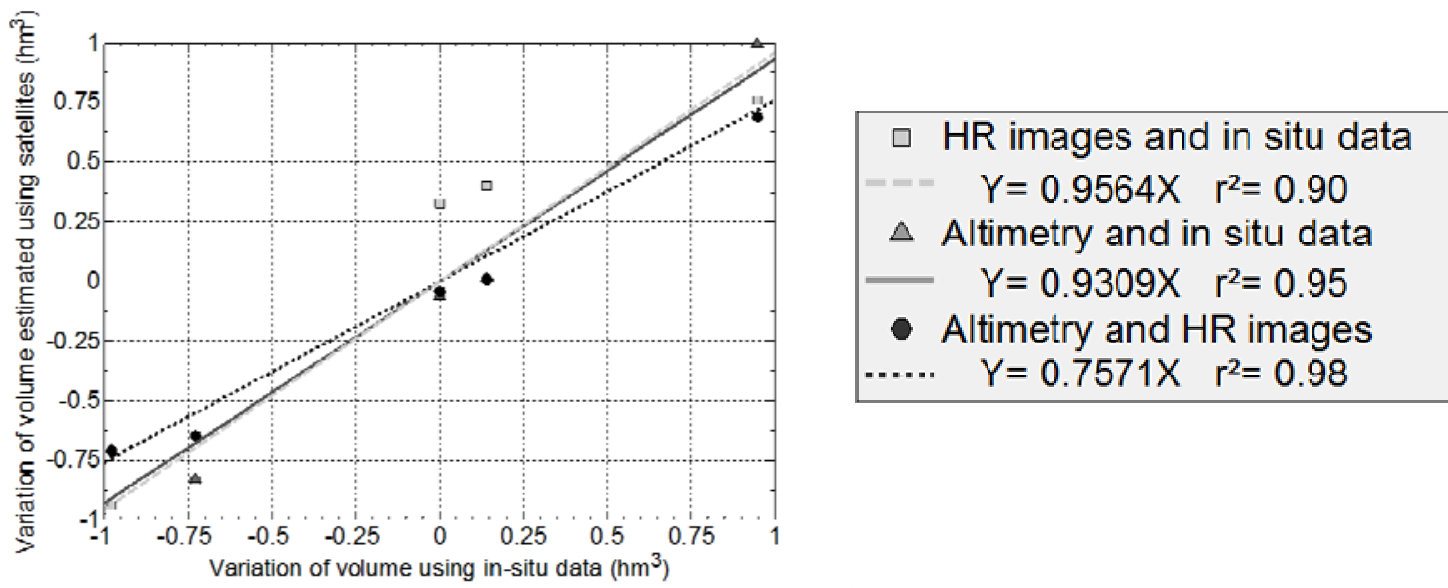

Figure 6. Variations de volume d'eau du lac estimées à partir des mesures in situ en abscisses, de l'imagerie HR et des données in situ (carrés gris clair), de l'altimétrie et des données in situ (triangles gris foncé) et par imagerie HR et altimétrie satellitaire (ronds noirs), en ordonnées.

En raison du faible nombre de données in situ disponibles, c'est la troisième méthode qui présente le plus d'intérêt dans le futur, même si elle ne fournit que les variations de volume d'eau. Les principaux inconvénients actuels de cette technique sont la faible couverture des traces altimétriques sous les tropiques et à moyenne latitude et la répétitivité temporelle en un même point comprise entre 10 et 35 jours. Avec le lancement dans les prochaines années de missions équipées des nouvelles générations de capteurs altimétriques SAR (Sentinel-3, Jason-CS) et InSAR (SWOT) et des futurs imageurs HR (Sentinel-1 and 2, Spot 7, Radarsat constellation, Alos-2), ces approches sont susceptibles d'être généralisées, fournissant ainsi un suivi plus complet et détaillé des eaux de surface. L'intérêt de ces nouveaux instruments est double. Le premier est lié à l'accroissement de la taille des fauchées des imageurs HR : un plus grand nombre de lacs sera compris au cours d'un passage de satellite. Le second concerne les nouveaux capteurs altimétriques. Les altimètres utilisant des technologies SAR et InSAR fourniront des élévations plus seulement le long de la trace, mais dans des fauchées allant de quelques à une centaine de kilomètres. Les résolutions spatiales seront ainsi améliorées, mais aussi les résolutions temporelles car un même lac pourra se trouver dans la fauchée de plusieurs altimètres. Les lacs ayant une intersection avec une trace altimétrique sur des distances de plus de 200 à 300 m, des variations de niveau d'eau supérieures à la précision des altimètres actuels, c'est-à-dire les lacs ayant des variations annuelles d'amplitude supérieures à quelques dizaines de centimètres), et dont la superficie est supérieure à $0,04 \mathrm{~km}^{2}$, devraient être détectés par les altimètres et les imageurs HR. 


\section{XIII ${ }^{\text {èmes }}$ Journées Nationales Génie Côtier - Génie Civil \\ Dunkerque, 2-4 juillet 2014}

\section{Conclusions}

Les trois approches présentées dans cette étude ont démontré un très fort potentiel pour le suivi des volumes d'eau et de leurs variations pour des lacs et réservoirs de petite taille comme ceux utilisés pour l'irrigation. Une bathymétrie du lac a été réalisée en avril 2014 dans le cadre du projet DRONEO. Elle sera utilisée pour définir de nouvelles relations hauteur-volume et valider les données de la mission altimétrique franco-indien (CNES-ISRO) AltiKa mise en orbite en février 2013. Les principales limitations de ces techniques sont relatives: (i) à la nécessité de disposer d'une courbe de tarage permettant de relier la mesure satellitaire au volume du lac, (ii) à la faible couverture spatiale des altimètres qui ne survolent à l'heure actuelle qu'un nombre limité de lacs, (iii) à la faible répétitivité des mesures spatiales, doublement pénalisante dans le cas de la synergie multi-technique. Une partie de ces limitations devraient disparaître avec la mise en orbite de futures missions satellitaires comme la série des Sentinel de l'ESA dès 2015 et surtout de l'altimètre franco-américain (CNES-NASA) Surface Water and Ocean Topography (SWOT) en 2020.

\section{Références bibliographiques}

ALSDORF D.E., RODRIGUEZ E., LETTENMAIER D.P. (2007). Measuring surface water from space. Review of Geophysics, Vol. 45, pp RG2002. http://dx.doi.org/10.1029/2006RG000197

BAUP F., FIEUZAL R., MARAIS-SICRE C., DEJOUX J-F., LE DANTEC V., MORDELET P., CLAVERIE M., HAGOLLE O., LOPES A., KERAVEC P., CESCHIA E., MIALON A., KIDD R. (2012). MCM'10: An experiment for satellite multi-sensors crop monitoring from high to low resolution observations. Geoscience and Remote Sensing Symposium, IEEE International, pp 4849-4852. http://dx.doi.org/10.1109/IGARSS.2012.6352527

BREIT H., FRITZ T., BALSS U., LACHAISE M., NIEDERMEIER A., VONAVKA M. (2010). TerraSAR-X SAR processing and products. IEEE Transactions on Geoscience and Remote Sensing, Vol. 48(2), pp 727-740. http://dx.doi.org/10.1109/TGRS.2009.2035497

CHERN J.S., LING J., WENG S.L. (2008). Taiwan's second remote sensing satellite. Acta Astronautica, Vol. 63(11), pp 1305-1311, http://dx.doi.org/ 10.1016/j.actaastro.2008.05.022 HAGOLLE O., HUC M., PASCUAL D. V., DEDIEU G. (2010). A multi-temporal method for cloud detection, applied to FORMOSAT-2, VENUS, LANDSAT and SENTINEL-2 images, Remote Sensing of Environment, 114, 1747-1755. http://dx.doi.org/10.1016/j.rse.2010.03.002

DUAN Z., BASTIAANSSEN W.G.M. (2013). Estimating water volume variations in lakes and reservoirs from four operational satellite altimetry databases and satellite imagery data. Remote Sensing of Environment, Vol. 134, pp 403-416. http://dx.doi.org/10.1016/j.rse.2013.03.010 
Thème 3 - Instrumentation, mesures, imagerie et télédétection

FRAPPART F., CALMANT S., CAUHOPE M., SEYLER F., CAZENAVE A. (2006). Preliminary results of ENVISAT RA-2 derived water levels validation over the Amazon basin. Remote Sensing of Environment, Vol. 100(2), pp 252-264. http://dx.doi.org/10.1016/j.rse.2005.10.027

FRAPPART F., PAPA F., SANTOS DA SILVA J., RAMILLIEN G., PRIGENT C., SEYLER F., CALMANT S. (2012). Surface freshwater storage and dynamics in the Amazon basin during the 2005 exceptional drought. Environment Research Letters, Vol. 7(4), pp 044010. http://dx.doi.org/10.1088/1748-9326/7/4/044010

MORENA L.C., JAMES K.V., BECK J. (2004). An introduction to the RADARSAT-2 mission. Canadian Journal of Remote Sensing, Vol. 30(3), pp 221-234. http://dx.doi.org/10.5589/m04-004

OECD (2012). Water Quality and Agriculture: Meeting the Policy Challenge. OECD Publishing, Paris, 160 p.

RICHARDS J.A. (1999). Remote Sensing Digital Image Analysis. Springer-Verlag, Berlin, 240 p.

RICKO M., BIRKETT C., CARTON J.A., CRETAUX J-F. (2012). Intercomparison and validation of continental water level products derived from satellite radar altimetry. Journal of Applied Remote Sensing, Vol. 6(1), pp 061710-061710. http://dx.doi.org/10.1117/1.JRS.6.061710

SAND A., DE BOISSEZON H. (2006). Reference remote sensing data bases: temporal series of calibrated and ortho- rectified satellite images for scientific use. 2nd International Symposium on Recent Advances in Quantitative Remote Sensing, Torrent, Spain.

SANTOS DA SILVA J., CALMANT S., SEYLER F., CORREA ROTUNO FILHO O.C., COCHONNEAU G., MANSUR W.J. (2010). Water levels in the Amazon basin derived from the ERS 2 and ENVISAT radar altimetry missions. Remote Sensing of Environment, Vol. 114(10), pp 2160-2181. http://dx.doi.org/j.rse.2010.04.020

TAUBE C.M. (2000). Chapter 12 : Three Methods for Computing the Volume of a Lake. Manual of Fisheries Survey Methods: with periodic updates. Michigan Department of Natural Resources, Fisheries Special Report 25, Ann Arbor, pp 1- 6. YOUNGER P. (2012). Water: All that matters. Hodder \& Stoughton, Londres, 160 p. ZELLI C. (1999). ENVISAT RA-2 advanced radar altimeter: Instrument design and pre-launch performance assessment review. Acta Astronautica, Vol. 44, pp 323- 333. http://dx.doi.org/10.1016/S0094-5765(99)00063-6 